\title{
Evidencias innegables de la halocinesis en la Cuenca Huallaga - Perú
}

\author{
Undeniable evidence of halokinesis in the Huallaga Basin - Peru
}

\author{
Jimmy Cotrina Cubas ${ }^{1}$
}

Recibido: 08/02/2021 - Aprobado: 13/07/2021 - Publicado: 23/12/2021

\section{RESUMEN}

La cuenca Huallaga ha sido estudiada e interpretada tectónicamente como una típica faja plegada y corrida de lámina delgada, sin embargo, el presente estudio nos ha llevado a la conclusión de que esta región ha experimentado características clásicas de halocinesis, la mayoría de las cuales se desarrollaron en el Jurásico sin relación con ningún evento tectónico compresional. Evidenciado por los numerosos afloramientos de domos salinos, descritos y cartografiados a lo largo del subandino, y con la interpretación de los datos sísmicos 2D que fueron registrados por diversas compañias petroleras. Finalmente, se ha puesto especial énfasis en el análisis de aquellos factores claves que permiten explicar el movimiento de la sal, su geometría y deformación de las estructuras reconocidas e interpretadas en las secciones sísmicas.

Palabras claves: Domos salinos; halocinesis; sal alóctona; sal autóctona; tectónica salina.

\begin{abstract}
The Huallaga basin has been studied and tectonically interpreted as typical fold and thrust belt of thin skin, however, the present study led us to the conclusion that this region had experimented the classic characteristics of halokinesis, the majorities of which had developed in the Jurassic unrelated to any compressional tectonic events. Evidenced by the numerous outcrops from the salt domes, described and mapped along the sub-Andean and with interpretation of 2D seismic acquired by diverse petroleum companies. Finally, special emphasis has been placed on the analysis of those key factors that explain the movement of salt, geometry and deformation of the different structures recognized and interpreted in seismic sections.
\end{abstract}

Keywords: Salt domes; halokinesis; allochthonous salt; autochthonous salt; salt tectonics. 


\section{INTRODUCCIÓN}

La cuenca Huallaga pertenece al sistema de cuencas subandinas y amazónicas de los Andes del norte del Perú. Los cartografiados geológicos regionales y diversas columnas litoestratigráficas describen aproximadamente +/- 8000 $\mathrm{m}$ de rocas sedimentarias de edades terciarias, cretácicas y pre-cretácicas y está caracterizado principalmente por la presencia de numerosos afloramientos de domos salinos. Paradójicamente, esta gran área rica en rocas evaporíticas se ha interpretado estructuralmente por una tectónica de faja plegada y corrida y se ha explorado solo desde ese punto de vista. Sin embargo, se ha realizado nuevas investigaciones morfoestructurales que lleven a postular que esta región ha sufrido características clásicas de la tectónica salina (Sempere \& Cotrina, 2018).

\subsection{Ubicación}

La cuenca Huallaga es una de las cuencas subandinas más extensas que cubre aproximadamente 5 millones de acres. Se desarrolla en la parte Norte-central del Perú entre los $6^{\circ} 5^{\prime}$ y $7^{\circ} 5^{\prime}$ de Latitud Sur y $75^{\circ} 30^{\prime}$ y $77^{\circ} 30^{\prime}$ de Longitud Oeste. Está limitada hacia el Norte con la Cuenca Santiago, al Oeste por la Cordillera Oriental, por el Noroeste con la Cuenca Marañón y hacia el Sureste con la Cuenca Ucayali. Políticamente, se ubica en el Departamento de San Martín en la región nororiental del territorio peruano, que es atravesado de sur a norte por el río Huallaga. (Figura 1).

\subsection{Estratigrafía}

Las unidades litoestratigráficas identificadas en afloramientos rocosos se describe en base a una columna geológica generalizada de la zona de estudio (Figura 2). Comprenden secuencias que van desde el Jurásico hasta la actualidad y están representadas por rocas del Grupo
Pucará (Jurásico inferior), Formación Sarayaquillo (Jurásico medio-superior), Grupo Oriente conformado por la Formación Cushabatay, Raya y Agua Caliente (Cretácico inferior), formaciones Chonta, Vivian y Cachiyacu (Cretácico superior), formaciones Huchpayacu, Casablanca, Yahuarango y Pozo (Paleógeno) y formación Chambira (Neógeno), (Sánchez, A. ; Chira, J. ; Valencia, 1997).

La geología se encuentra bien expuesta en las montañas (corte de carreteras), valles y quebradas, mientras que en la llanura amazónica los afloramientos son esporádicos y los terrenos están cubiertos por depósitos aluviales.

\section{MÉTODOS}

El presente estudio se ha realizado utilizando artículos, publicaciones, informes técnicos geológicos y datos sísmicos 2D, que se encuentran disponibles en el Banco de Datos de PERUPETRO S.A, el cual ha permitido la interpretación sismo-estratigráfica como soporte y un mejor entendimiento de la geología del subsuelo.

La metodología utilizada se ha basado en la integración de los datos de geología de superficie y de subsuelo. Para realizar la calibración y análisis de amarre sísmica - pozo, se generaron sismogramas sintéticos en los pozos Ponasillo 1X, Loreto 1X, Shanusi 1X y Yurimaguas 1X. Para cada uno de los pozos se utilizaron los registros eléctricos sónico, densidad y check shots previamente calibradas con el objetivo de calcular el coeficiente de reflexión e impedancias acústicas. finalmente se combinó con una ondícula extraída (Ricker de $25 \mathrm{hz}$ ) para producir una respuesta sísmica a lo largo del pozo obteniendo con esta un buen amarre entre la sísmica y el sismograma.
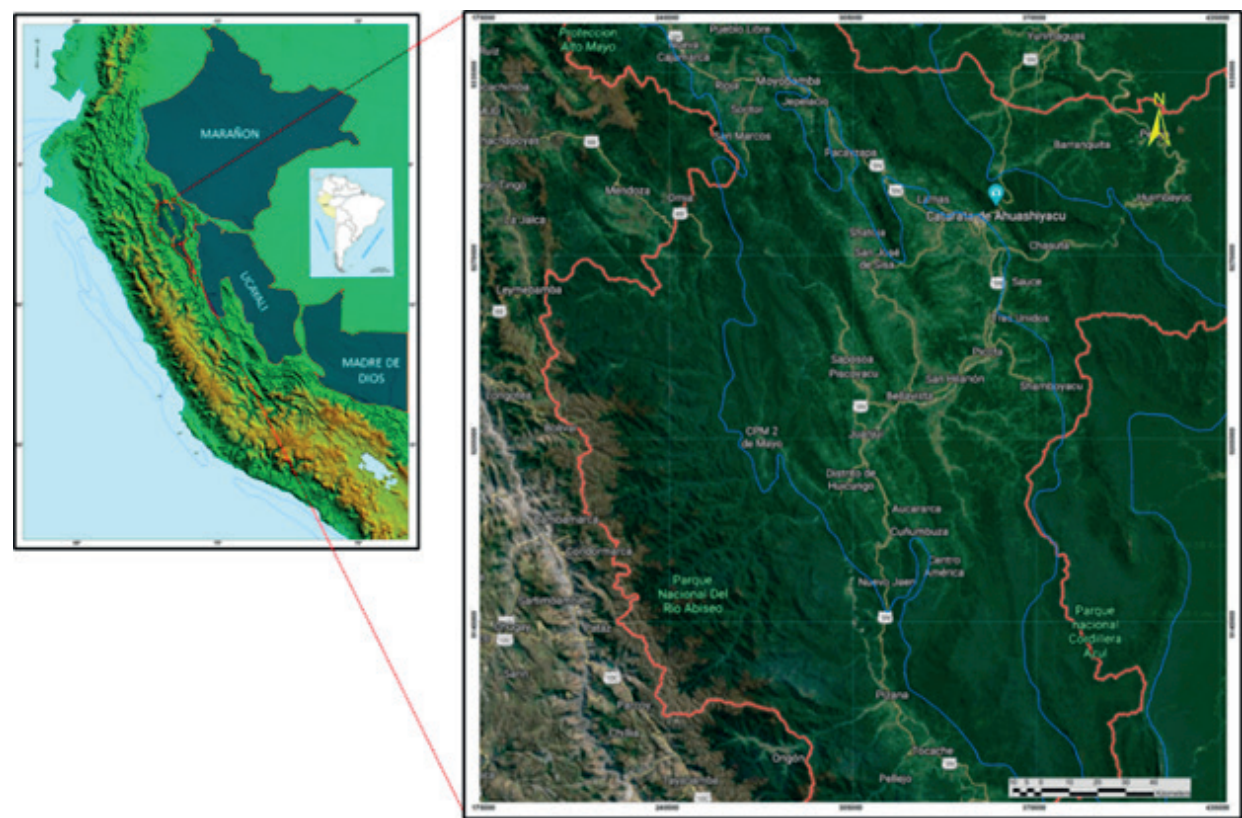

Figura 1. Imagen satelital, se aprecia el área de estudio la cuenca Huallaga (borde azul) ubicada en la región San Martin. Elaboración propia. 


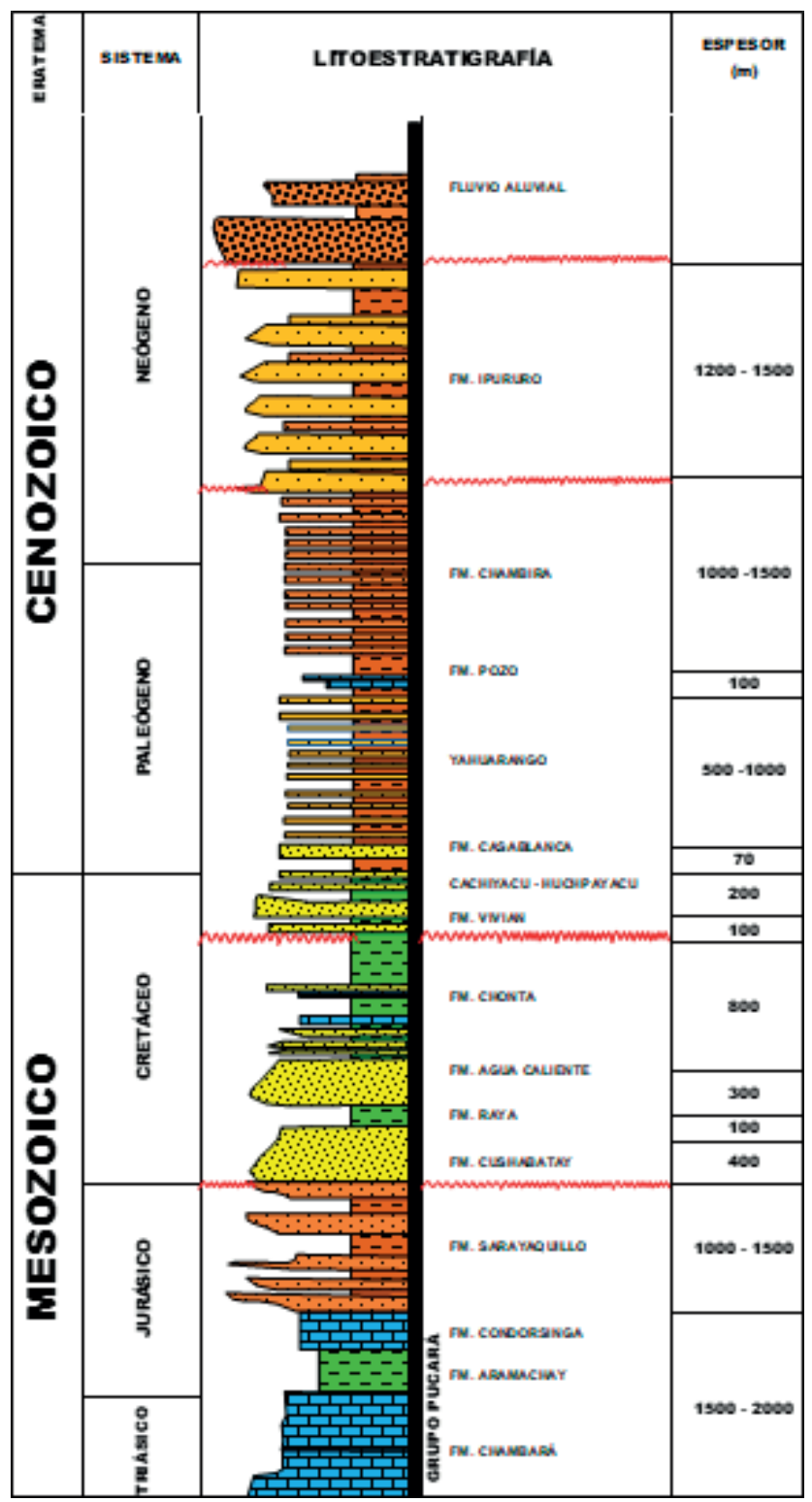

Figura 2. Columna generalizada de la cuenca Huallaga (Perupetro, 2018)

\section{RESULTADOS Y DISCUSIÓN}

\subsection{Afloramientos y génesis de la sal}

Grandes domos de sal surgen al este de la Cordillera Oriental, extendiéndose desde la frontera con Ecuador hasta al menos los $12^{\circ} \mathrm{S}$ de latitud, cubriendo ampliamente las cuencas de Santiago, Huallaga, Marañón occidental, Pachitea, Ene, y áreas adyacentes al oeste (Figura 3). De igual manera la ocurrencia de domos de sal es apreciados en las tierras altas andinas, en el centro de Perú y hacia el sureste hasta la región de Cusco.

Los datos son consistentes, el periodo de interés comenzó con el inicio del desarrollo generalizado de un rift de trasarco hace $245 \mathrm{Ma}$ y continuó hasta $\sim 88 \mathrm{Ma}$. La deformación extensional fue inicialmente dramática (Grupo Mitu), generando áreas deprimidas (hemigrábenes) que fueron invadidas por el agua de mar con niveles altos de evaporación (Figura 4). Se depositaron centenares de metros de sal (Triásico Superior) como una unidad estratigráfica (con variaciones de espesores), definida como formación Pareni, que originalmente cubría al Grupo Mitu típico (rocas volcánicas y lechos rojos continentales) y subyace al Grupo Pucará (carbonatos marinos), (Sempere \& Cotrina, 2018).

La sucesión Mitu-Pareni-Pucará registra el desarrollo de un sistema de fallamientos, cuyo eje probablemente corría a lo largo de la actual Cordillera Oriental y la acumulación de grandes cantidades de sal preservados en hemigrábenes. Debido a que el Grupo Pucará suprayacente (Triásico-Liásico) consiste principalmente en carbonatos de $>1 \mathrm{~km}$ de espesor es probable que la Sal de Pareni sufriera halocinesis tan pronto como el Liásico tardío se depositó (Sempere \& Cotrina, 2018) afectando a los niveles sobreyacentes (Figura 5). 

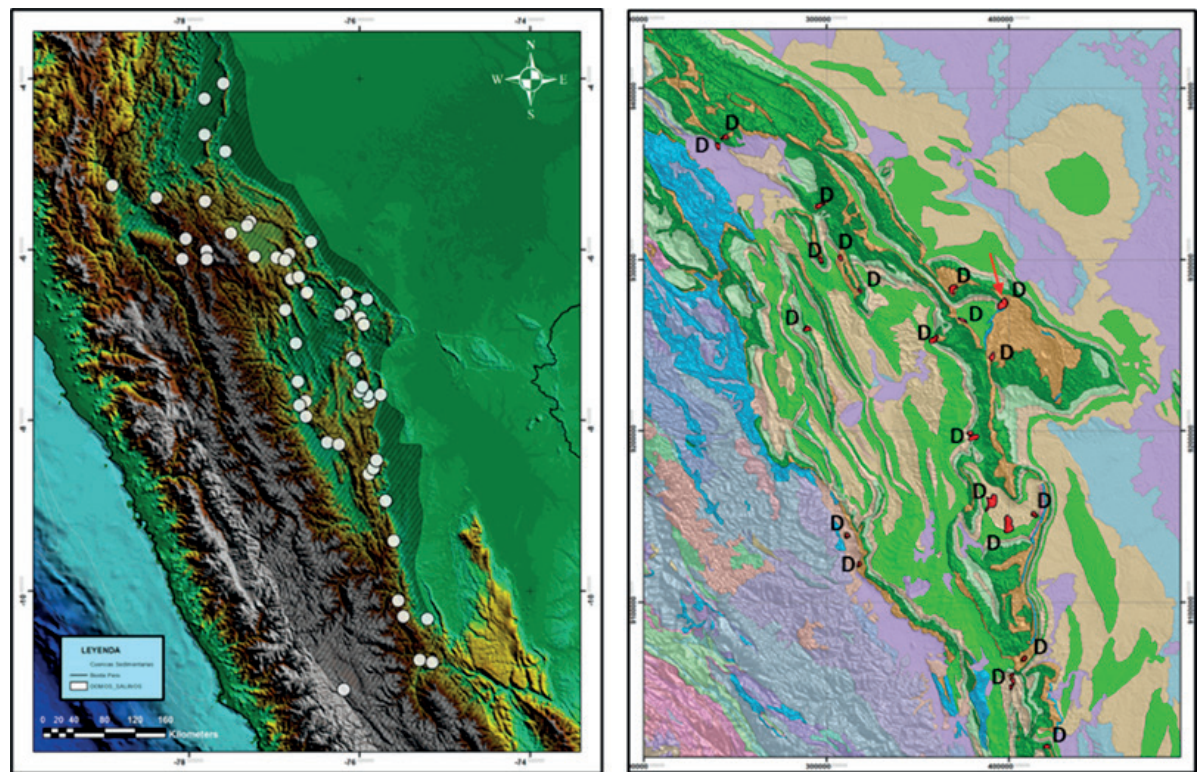

Figura 3. Mapa de afloramientos de domos salinos en la faja Subandina

(Sempere \& Cotrina, 2018)

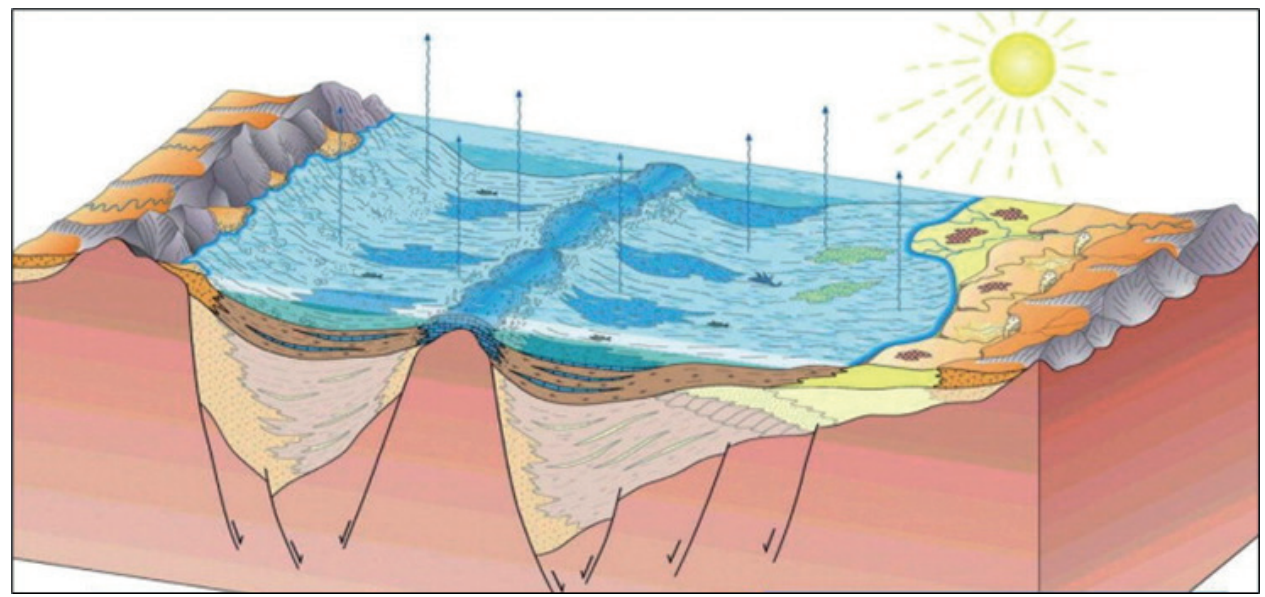

Figura 4. Ingreso progresivo del mar del Pucará en un clima árido y cálido que provocaron niveles altos de evaporación y depósitos de sal preservados en hemigrábenes (Gomes et al., 2012)

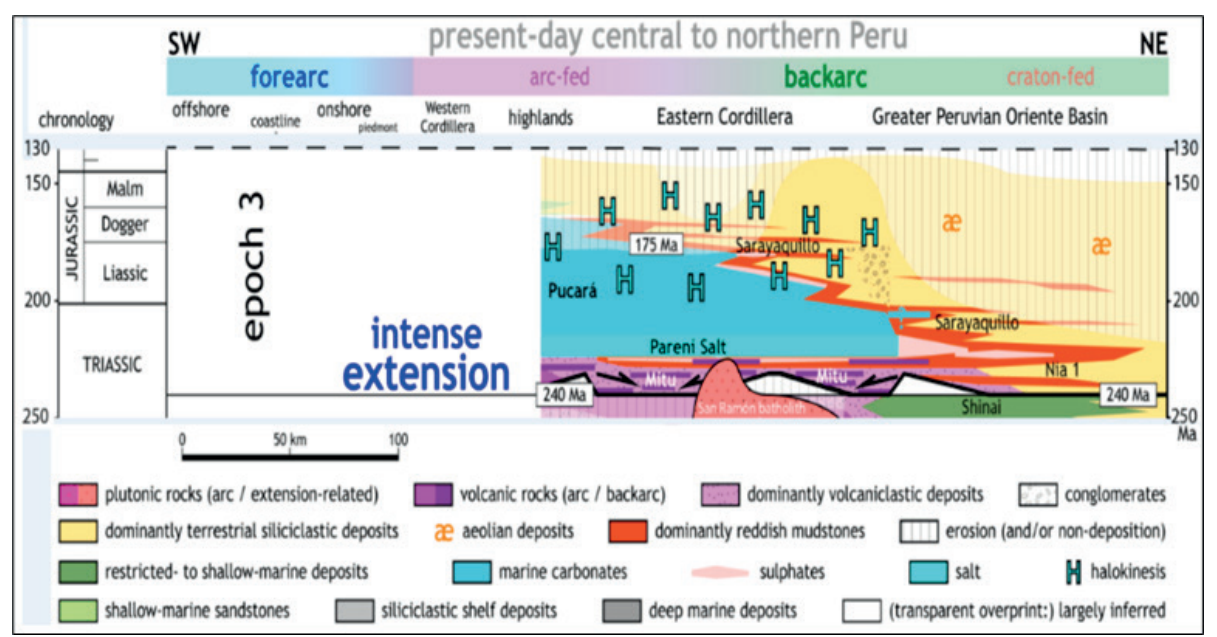

Figura 5. Transecto estratigráfico generalizado del centro y norte del Perú $\left(\sim 8-11^{\circ} \mathrm{S}\right)$ desde 240 Ma hasta $\sim 130 \mathrm{Ma}$ (Fiestas et al., 2019) 


\subsection{Interpretación sismo-estratigráfica}

Se ha evidenciado a través de la interpretación sísmica 2D la presencia de dos niveles de sal bien diferenciados. La primera se ubica hacia la parte inferior (sal Autóctona) quienes fueron depositadas en los hemigrábenes durante la entrada del mar de Pucará ( 222-212 Ma). La segunda se ubica hacia la parte superior (sal alóctona) de las líneas símicas, son aquellas que se han removilizado, emplazado y deformando a las Formaciones suprayacentes. Asimismo, se evidencia un mayor desarrollo en espesor de rocas terciarias hacia el Suroeste de la cuenca con respecto a la zona central, debido a la migración y disolución de la sal (sal soldada), generando una zona cóncava (sinclinal), mientras en la en la parte central tenemos estratos subparalelos (Figura 6 y Figura 7). Las soldaduras de sal también son de gran importancia en la industria de los hidrocarburos porque pueden proporcionar un sello hidrodinámico y entrampan hidrocarburos o podrían permitir la transmisión de fluidos de la roca madre al reservorio (Jackson et al., 2014). Finalmente, se aprecia geometrías como estratos de crecimientos y discordancias angulares (Figura 7) generados por la migración y emplazamiento de los cuerpos salinos (Figura 8) durante la deposición de los sedimentos, todos ellos son características típicas de la halocinesis en la cuenca.

Es importante recordar que, la halocinesis se desencadena cuando la carga sedimentaria que cubre los depósitos salinos alcanza una densidad superior a la densidad de la halita $(\sim 2,2 \mathrm{~g} / \mathrm{cm})$ por lo general ocurre

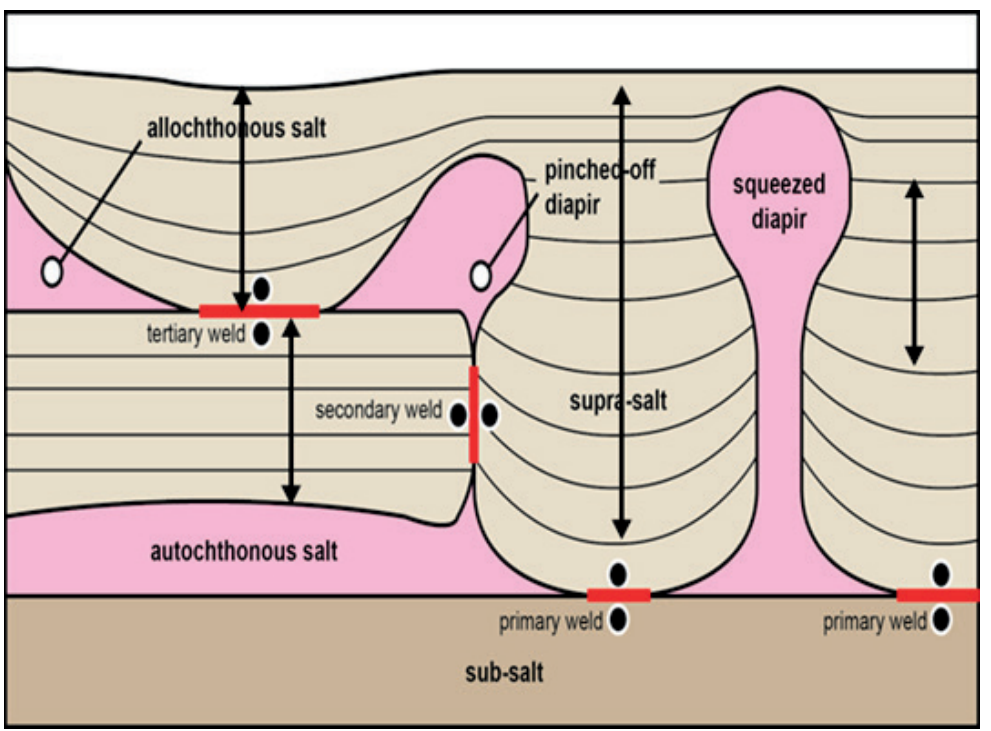

Figura 6. Representación esquemática de la sal soldada, autóctona y alóctona y su relación con la variación de espesores (Jackson et al., 2014)

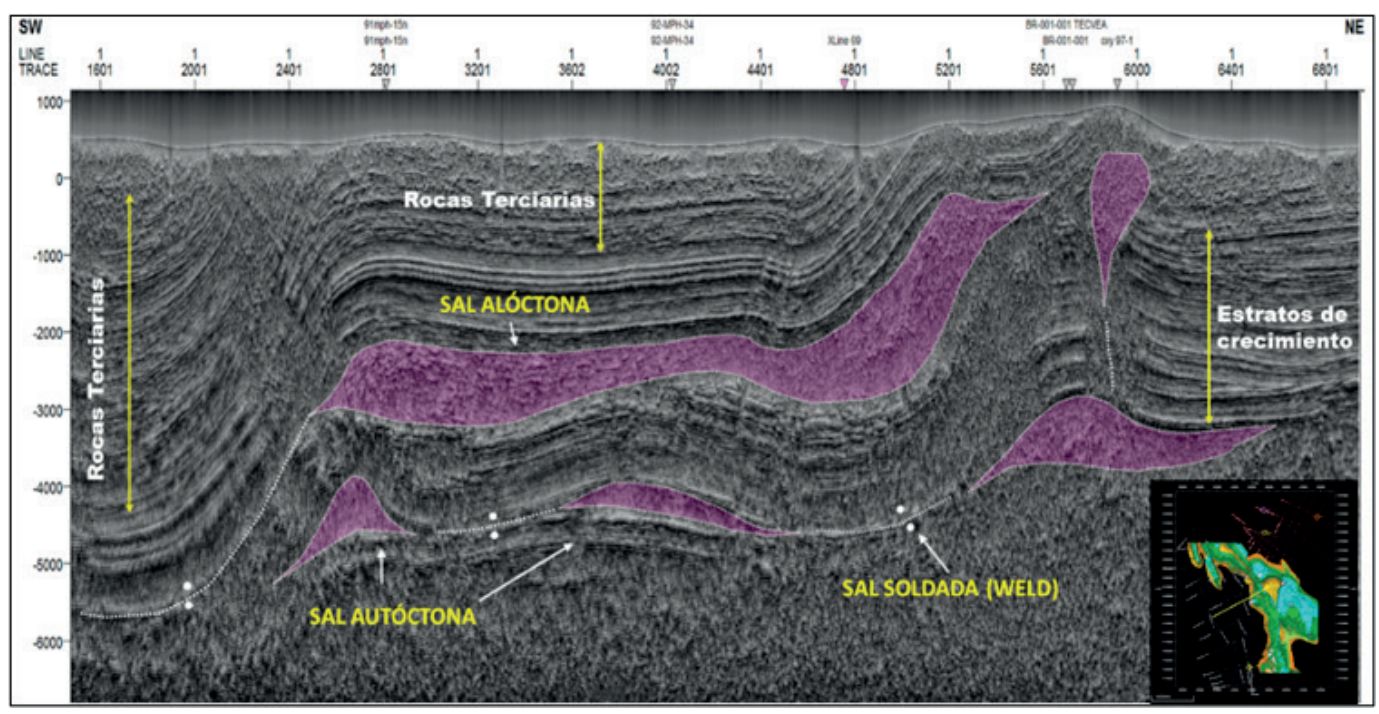

Figura 7. Se aprecia en la Línea Símica 91-MPH-23 dos niveles de sal bien diferenciados la inferior sal autóctona y una superior sal alóctona. Asimismo, un mayor desarrollo de rocas terciarias hacia el suroeste de la cuenca con respecto a la parte central.

Elaboración propia. 


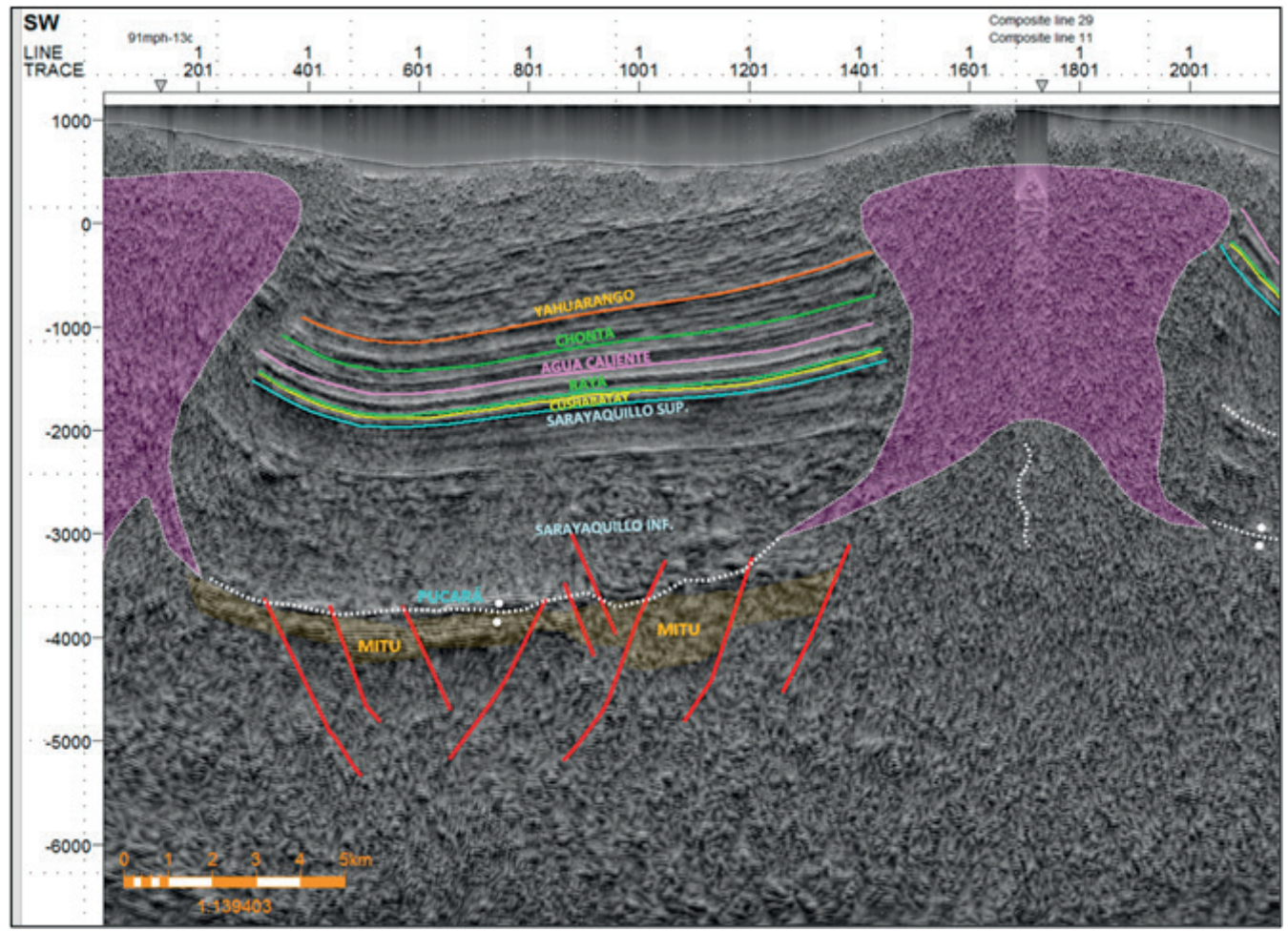

Figura 8. Se aprecia la Línea Sísmica 91-MPH-25 la geometría de los domos salinos atravesando a toda la columna estratigráfica, a consecuencia de la halocinesis. Elaboración propia.

cuando los sedimentos suprayacentes alcanzan un espesor aproximado de $1.5 \mathrm{~km}$ (Figura 9). Bajo este concepto, diversos cuerpos salinos que han sido identificados en el área de estudio a través de la interpretación sísmica 2D y los afloramientos rocosos indican consistentemente una tectónica salina progresiva durante la deposición de la formación Sarayaquillo (explicación a la variación de espesor de esta unidad estratigráfica) e influenciado por la reactivación de la extensión jurásica $(\sim 175-88 \mathrm{Ma})$. ver Figura 10.

\section{CONCLUSIONES}

- Diversos afloramientos de domos salinos en superficie a lo largo de la cuenca Huallaga se encuentran atravesando a toda la columna estratigráfica y se encuentran íntimamente relacionados a procesos de halocinesis.

- La interpretación sismo-estratigráfica $2 \mathrm{D}$, ha permitido reconocer dos niveles de sal bien diferenciados en la cuenca Huallaga, la inferior (sal alóctona) y la superior (sal autóctona), el cual evidencia una migración lateral de la sal y su redistribución en una sucesión y geometrías de cuerpos salinos.

- La identificación de geometrías como estratos de crecimientos y discordancias angulares originados por la migración y emplazamiento de los cuerpos salinos durante la deposición de los sedimentos son características típicas de halocinesis en la cuenca Huallaga.

- La halocinesis se desencadena cuando la carga sedimentaria que cubre los depósitos salinos, alcanza una densidad superior a la densidad de la halita $(\sim 2,2 \mathrm{~g} / \mathrm{cm})$ por lo general ocurre cuando los estratos suprayacentes alcanzan un espesor aproximado de $1.5 \mathrm{~km}$. Bajo este concepto, se ha identificado una tectónica salina progresiva durante la deposición de la formación Sarayaquillo (explicación a la variación de espesor de esta unidad estratigráfica).

- La deformación extensional durante la deposición de los sedimentos del Grupo Mitu, generaron áreas deprimidas (hemigrábenes) que fueron invadidas posteriormente por el Ingreso progresivo del mar del Pucará ( 222-212 Ma) en un clima árido y cálido que provocaron niveles altos de evaporación y e ingentes depósitos de sal.

\section{AGRADECIMIENTOS}

Especial agradecimientos al Dr. Thierry Sempere por compartir sus conocimientos y experiencias en Tectónica Salina. A Asaid Bandach por haber depositado su confianza en el liderazgo de los proyectos técnicos de exploración y desarrollo de hidrocarburos (2015-2018). A PERUPETRO S.A, por permitir el acceso a los datos e información públicos para la realización de esta investigación. 


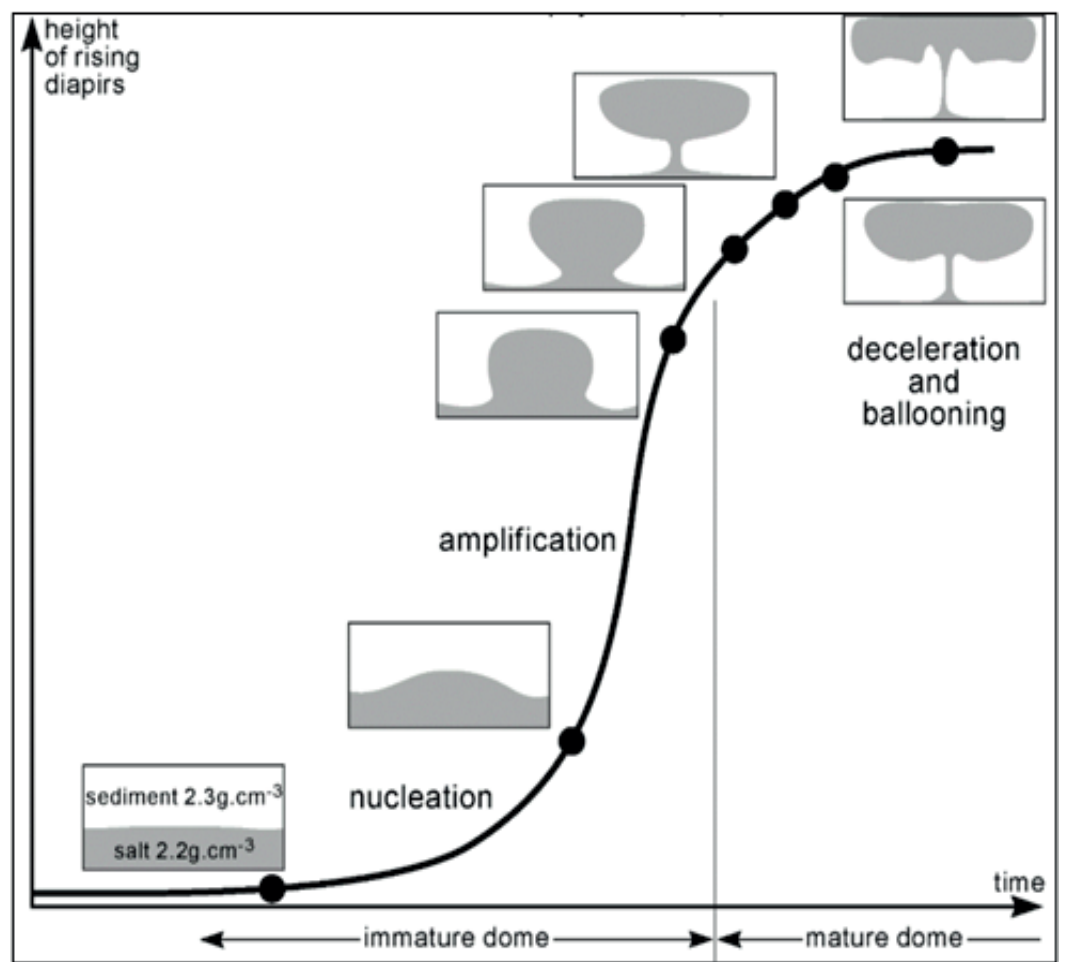

Figura 9. Se aprecia que las estructuras diapíricas geológicas son impulsadas hacia arriba en su totalidad por fuerzas de flotación que resultan del contraste de densidad entre una capa de baja densidad y las rocas más pesadas que se encuentran sobre ella (Román Berdiel \& Santolaria Otín, 2012)

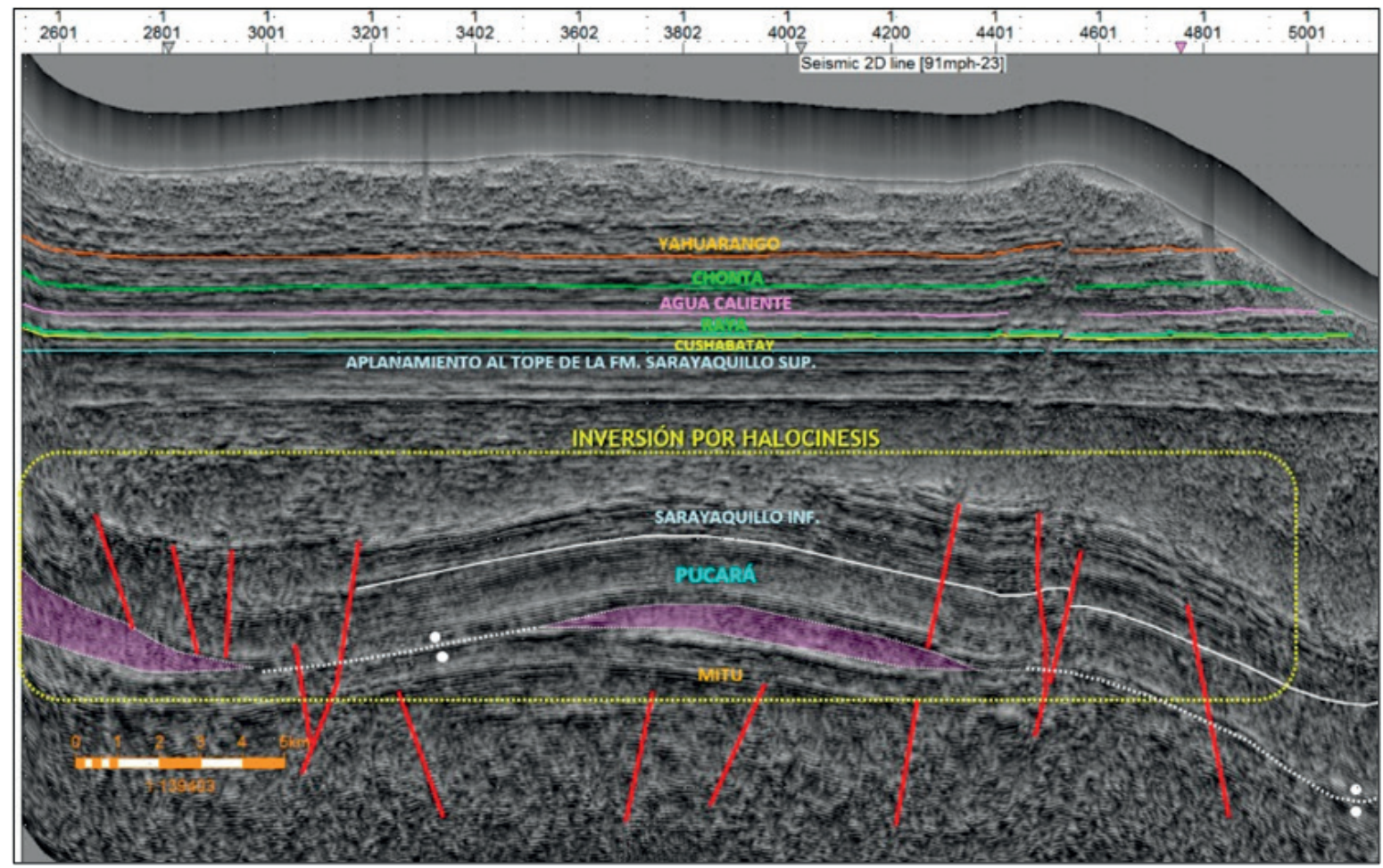

Figura 10. Aplanamiento al tope de la formación Sarayaquillo en las secciones sísmica 91-MPH-23, se evidencia estructuraciones pre-cretácicas por halocinesis. Elaboración propia. 


\section{REFERENCIAS}

Fiestas, J., Berrospi, R., Leach, D., \& Sempere, T. (2019). Tectónica salina e hidrocarburos como guías de exploración de yacimientos MVT : $\mathrm{Zn}-\mathrm{Pb}$ en los Andes del Perú central. ProEXPLO 2019, 1-6. https://app.ingemmet.gob.pe/ biblioteca/pdf/Lib-122-296.pdf

Gomes, P. O., Kilsdonk, B., Grow, T., Minken, J., \& Barragan, R. (2012). Tectonic evolution of the outer high of Santos Basin, Southern São Paulo Plateau, Brazil, and implications for hydrocarbon exploration. In AAPG Memoir (Issue 100, pp. 125-142). https://doi.org/10.1306/13351550M1003530

Jackson, C. A. L., Rodriguez, C. R., Rotevatn, A., \& Bell, R. E. (2014). Geological and geophysical expression of a primary salt weld: An example from the Santos Basin, Brazil. Interpretation, 2(4), SM77-SM89. https://doi.org/10.1190/ INT-2014-0066.1

Perupetro. (2018). Huallaga basin petroleum system. https://www.perupetro.com.pe/wps/wcm/ connect/corporativo/469bffbe-b3f $2-42$ b0 0 -baff$\mathrm{fbf7ff} 4 \mathrm{df} 270 / \mathrm{MEMORIA}+\mathrm{PERUPETRO}+2018 \mathrm{a}$. pdf?MOD=AJPERES\&MemoriaAnual2018
Román Berdiel, T., \& Santolaria Otín, P. (2012). Cómo reproducir diapiros en el aula. Enseñanza de Las Ciencias de La Tierra: Revista de La Asociación Española Para La Enseñanza de Las Ciencias de La Tierra, 20(3), 262-269. https:// www.researchgate.net/publication/257295364 Como reproducir_diapiros_en_el_aula_Playing_diapirs_in_the_ classroom

Sánchez, A. ; Chira, J. ; Valencia, M. (1997). Geología De Los Cuadrángulos De Tarapoto , Papa Playa , Utcurarca Y Yanayacu. Carta Geológica Nacional, Serie $A(94), 264$. https://repositorio.ingemmet.gob.pe/ handle/20.500.12544/51

Sempere, T., \& Cotrina, J. (2018). An overlooked giant salt basin in peru. Ix Ingepet 2018, 9, 1-18. https://es.scribd.com/ document/396237433/SempereCotrina-2018-INGEPETFinal-Lite 